\title{
THE CHARACTERISTIC ROOTS OF THE PRODUCT OF TWO MATRICES
}

\author{
N. A. KHAN \\ (Received January. 20,1957)
}

1. Introduction and notations. Let $A$ be a square matrix of order $n$ with elements belonging to the field of complex numbers. Further, let $c(A)$ stand for an arbitrary characteristic root of $A$, whereas $\bar{c}(A)$ denotes the complex conjugate of $c(A)$.

In a recent paper [2], this author has found the upper bound for an arbitrary characteristic root $c(A B)$ of the product of two matrices $A$ and $B$ in terms of their elements. The purpose of this paper is to find the upper bounds for the real and imaginary parts of $c(A B)$ in terms of the elements of the associated Hermitian matrices $\left(A+\overline{A^{\prime}}\right) / 2,\left(A-A^{\prime}\right) / 2 i,\left(B+\bar{B}^{\prime}\right) / 2$ and $\left(B-B^{\prime}\right) / 2 i$. In what follows, $R_{i}(A)$ will denote the sum of the absolute values of the elements of an arbitrary matrix $A$ in the $i$-th row, $T_{i}(A)$ will denote the sum of the absolute values of the elements of $A$ in the $i$-th column, and $R(A), T(A)$ will stand for the greatest of the $R_{i}(A)$ and $T_{t}(A)$ respectively.

2. Upper bounds for the real and imaginary parts of $\mathrm{c}(\mathbf{A B})$.

Theorem. Let $A$ and $B$ be two commuting $n$-square complex matrices. If $S_{r}^{\prime}(A), S_{\prime \prime}^{\prime \prime}(A), S_{\prime}^{\prime}(B), S_{, .}^{\prime \prime}(B)$ are the sums of the absolute values of the elements in the $r$-th row of $\left(A+\bar{A}^{\prime}\right) / 2,\left(A-\overline{A^{\prime}}\right) / 2 i,\left(B+\bar{B}^{\prime}\right) / 2,\left(B-\overline{B^{\prime}}\right) / 2 i$ respectively, and if $S^{\prime}(A), S^{\prime \prime}(A), S^{\prime}(B), S^{\prime \prime}(B)$ are respectively the greatest of the $S_{r}(A), S_{r}^{\prime \prime}(A), S_{s}^{\prime}(B), S_{r^{\prime \prime}}^{\prime \prime}(B)$, then

and

$$
\begin{aligned}
& \left|\frac{c(A B)+\bar{c}(A B)}{2}\right| \leqq S^{\prime}(A) S^{\prime}(B)+S^{\prime \prime}(A) S^{\prime \prime}(B), \\
& \left|\frac{c(A B)-\bar{c}(A B)}{2 i}\right| \leqq S^{\prime}(A) S^{\prime \prime}(B)+S^{\prime \prime}(A) S^{\prime}(B) .
\end{aligned}
$$

Proof. Any square matrix $A=\frac{A+\overline{A^{\prime}}}{2}+i \frac{A-A^{\prime}}{2 i}=P+i Q$, say, where $P=\left(p_{i j}\right), Q=\left(q_{i j}\right)$ are Hermitian matrices; and any square matrix $B=$ $\frac{B+\overline{B^{\prime}}}{2}+i \frac{B-\overline{B^{\prime}}}{2 i}=U+i V$, say, where $U=\left(u_{i j}\right)$ and $V=\left(v_{i j}\right)$ are Hermitian matrices. Thus

and

$$
\begin{aligned}
& A B=P U-Q V+i(P V+Q U), \\
& A^{\prime} B^{\prime}=P U-Q V-i(P V+Q U) .
\end{aligned}
$$

Now, if $\lambda$ is a characteristic root of $A B$, there exists a complex unit vector $x=\left(x_{1}, x_{2}, \ldots x_{n}\right)^{r}$, such that 


$$
\lambda x=A B x .
$$

Premultiplying the above equation by $\overline{x^{\prime}}$, we have

$$
\begin{gathered}
\lambda \overline{x^{\prime}} x=\overline{x^{\prime}} A B x, \\
\lambda=\overline{x^{\prime}} A B x .
\end{gathered}
$$

or,

Taking the conjugate transpose of (5) we have

$$
\begin{aligned}
\bar{\lambda} & =\overline{x^{\prime}}\left(B^{\prime} A^{\prime}\right) x \\
& =\bar{x}^{\prime}\left(A^{\prime} B^{\prime}\right) x,
\end{aligned}
$$

since $A B=B A$ implies $A^{\prime} B^{\prime}=B^{\prime} A^{\prime}$.

From (5) and (6) by addition and subtraction, we have

$$
\frac{\lambda+\bar{\lambda}}{2}=x^{\prime}(P U-Q V) x
$$

and

$$
\frac{\lambda-\bar{\lambda}}{2 i}=x^{\prime}(P V+Q U) x \text {. }
$$

From (7) and (8) we determine the upper bounds for $\left|\frac{\lambda+\bar{\lambda}}{2}\right|$ and $\left|\frac{\lambda-\bar{\lambda}}{2 i}\right|$. Since these relations are identical in form, it is sufficient to carry the computation through one of them only.

Taking the absolute values in (7), we get

$$
\begin{aligned}
\left|\frac{\lambda+\bar{\lambda}}{2}\right| & =\left|x^{\prime}(P U-Q V) x\right| \\
& =\left|\Sigma_{r, s} \alpha_{r s} \overline{x_{r}} x_{s}-\Sigma_{r, s} \beta_{r s} \overline{x_{r}} \cdot x_{s}\right|
\end{aligned}
$$

where $\alpha_{r s}$ and $\beta_{r s}$ denote the elements of $P U$ and $Q V$, respectively, in the $(r, s)$-th position, or,

$$
\left|\frac{c(A B)+c(A B)}{2}\right| \leqq\left|\Sigma_{r, s} \alpha_{r \cdot s} x_{r} x_{s}\right|+\left|\Sigma_{r, s} \beta_{r s} x_{r} x_{s}\right| .
$$

Let $\xi_{r}=\left|x_{r}\right|$, so that $\sum_{r} \xi_{r}^{2}=1$ and $\xi_{r} \xi_{s} \leqq 1 / 2\left(\xi_{r}^{\prime}+\xi_{s}^{\prime}\right)$. Now, we consider the two terms on the right-hand side of (9) separately.

$$
\begin{aligned}
\left|\Sigma_{r, s} \alpha_{r s} x_{r} x_{s}\right| & \leqq \Sigma_{r, s}\left|\alpha_{s r}\right| \xi_{r} \xi_{s} \\
& \leqq 1 / 2 \sum_{r, s}\left|\alpha_{r s}\right|\left(\xi_{r}^{2}+\xi_{s}^{2}\right) \\
& =1 / 2\left\{\sum_{r} \xi_{r} \sum_{s}\left|\alpha_{r s}\right|+\sum_{s} \xi_{s} \sum_{r}\left|\alpha_{r s}\right|\right\} \\
& =1 / 2\left\{\sum_{r} \xi_{r}^{2} R_{r}(P U)+\sum_{s} \xi_{s}^{2} T_{s}(P U)\right\}
\end{aligned}
$$

Supposing that $R_{r}(P U)$ and $T_{s}(P U)$ attain their maximum values respectively for $r=h$ and $s=k$, we have

$$
\left|\sum_{r s} \alpha_{r s} \bar{x}_{r} x_{s}\right| \leqq 1 / 2\left\{R_{h l}(P U)+T_{k}(P U)\right\}
$$


But, by definition,

$$
\begin{aligned}
R_{h}(P U) & =\left|\sum_{s} p_{h s} u_{s 1}\right|+\left|\sum_{s} p_{h s} u_{s !}\right|+\ldots+\left|\sum_{s} p_{h s} u_{s n}\right| \\
& \leqq \sum_{s}\left|p_{h i s}\right|\left|u_{s 1}\right|+\sum_{s}\left|p_{h s}\right|\left|u_{s 2}\right|+\ldots+\sum_{s}\left|p_{h s}\right|\left|u_{s n}\right| \\
& =\left|p_{h 1}\right| \sum_{t}\left|u_{1 t}\right|+\left|p_{h 2}\right| \sum_{t}\left|u_{2 t}\right|+\ldots+\left|p_{h^{n}}\right| \sum_{t}\left|u_{n t}\right| \\
& =\left|p_{h 1}\right| R_{1}(U)+\left|p_{h 2}\right| R_{2}(U)+\ldots+\left|p_{h n}\right| R_{n}(U) \\
& \leqq R(U)\left(\left|p_{h 1}\right|+\left|p_{h 2}\right|+\ldots+\left|p_{h n}\right|\right) . \\
& =R_{h}(P) R(U) \\
& \leqq R(P) R(U)=S^{\prime}(A) S^{\prime}(B)
\end{aligned}
$$

$$
\text { and } \begin{aligned}
T_{k}(P U) & =\left|\sum_{s} p_{1 s} u_{s k}\right|+\left|\sum_{s} p_{2 s} u_{s k}\right|+\ldots+\left|\sum_{s} p_{n s} u_{s k}\right| \\
& \leqq \sum_{s}\left|p_{1 s}\right|\left|u_{s k}\right|+\sum_{s}\left|p_{2 s}\right|\left|u_{s k}\right|+\ldots+\sum_{s}\left|p_{n s}\right|\left|u_{s k}\right| \\
& =\left|u_{1 k}\right| \sum_{t}\left|p_{t 1}\right|+\left|u_{: k}\right| \sum_{t}\left|p_{t z}\right|+\ldots+\left|u_{n k}\right| \sum_{t}\left|p_{i n}\right| \\
& =\left|u_{2 k}\right| T_{1}(P)+\left|u_{i k}\right| T_{2}(P)+\ldots+\left|u_{n k}\right| T_{n}(P) \\
& \leqq T(P) T_{k}(U) \\
& \leqq T(P) T(U)=S^{\prime}(A) S^{\prime}(B)
\end{aligned}
$$

since for any Hermitian matrix $H=\left(h_{r s}\right), T(H)=\max _{s} T_{s}(H)=\max _{s} \sum_{r}\left|h_{r s}\right|$ $=\max _{s} \sum_{r}\left|h_{s r}\right|=\max _{s} R_{s}(H)=R(H)$.

The inequalities (10) and (11) give

$$
\left|\Sigma \alpha_{r s} \overline{x_{r}} x_{s}\right| \leqq S^{\prime}(A) S^{\prime}(B) \text {. }
$$

Similarly, taking $\left|\Sigma \beta_{r s} \bar{x}_{r} x_{s}\right|$ and proceeding as we did in establishing (12), we shall prove

$$
\left|\Sigma \beta_{r s} x_{r} x_{s}\right| \leqq R(Q) R(V)=S^{\prime \prime}(A) S^{\prime \prime}(B) .
$$

Combining (12) and (13), we obtain

$$
\left|\frac{c(A B)+\bar{c}(A B)}{2}\right| \leqq S^{\prime}(A) S^{\prime}(B)+S^{\prime \prime}(A) S^{\prime \prime}(B) .
$$

Similarly, starting with (8), we can establish the inequality (2).

This completes the proof of the Theorem.

The condition, that $A$ and $B$ commute, imposed on the matrices in the Theorem, is necessary as shown by the following example: 


$$
A=\left(\begin{array}{rr}
0 & i \\
2 i & 0
\end{array}\right), \quad B=\left(\begin{array}{cr}
0 & -2 i \\
-i & 0
\end{array}\right), \quad A B=\left(\begin{array}{ll}
1 & 0 \\
0 & 4
\end{array}\right) \neq B A .
$$

Here $S^{\prime}(A)=S^{\prime}(B)=1 / 2, S^{\prime \prime}(A)=S^{\prime \prime}(B)=3 / 2, c(A B)=1,4$, and 4 is not less than or equal to $5 / 2$.

3. Some particular cases of (1) and (2). (i). Let $A$ and $B$ be commuting $n$-square Hermitian matrices, so that $A B$ is also Hermitian and all $c(A B)$ are real. In this case $S^{\prime}(A)=R(A), S^{\prime}(B)=R(B)$, and $S^{\prime \prime}(A)=S^{\prime \prime}(B)=0$. Thus, for matrices $A$ and $B$ defined above, (1) reduces to

$$
|c(A B)| \leqq R(A) R(B)
$$

a result proved in [2].

(ii). Again, if $A$ and $B$ are commuting skew-Hermitian matrices of the same order, $A+\bar{A}^{\prime}=B+B^{\prime}=0,\left(A-\bar{A}^{\prime}\right) / 2 i=A / i$, and $\left(B-B^{\prime}\right) / 2 i=B / i$. Also $A B$ is Hermitian, so that all the characteristic roots of $A B$ are real, $S^{\prime}(A)=S^{\prime}(B)=0$ and $S^{\prime \prime}(A)=R(A / i)=R(A)$, and $S^{\prime \prime}(B)=R(B / i)=R(B)$. In this case also (10) reduces to

$$
|c(A B)| \leqq R(A) R(B) .
$$

(iii). Let us put $B=I$, for which $S^{\prime}(B)=1$ and $S^{\prime \prime}(B)=0$. In this case (1) and (2) reduce to

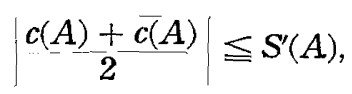

and

$$
\left|\frac{c(A)-c \overline{(A)}}{2 i}\right| \leqq S^{\prime \prime}(A)
$$

results due to E. T. Browne [1], and W. V. Parker [3], giving the upper bounds for the real and imaginary parts of an arbitrary characteristic root of $A$ in terms of the elements of the associated Hermitian matrices $\left(A+A^{\prime}\right) / 2$ and $\left(A-A^{\prime}\right) / 2 i$.

\section{REFERENCES}

[1] E.T.BRownE, The characteristic roots of a matrix, Bull. Amer. Math. Soc., 36(1930), 705-10.

[2] N.A. KHAN, The characteristic roots of the product of matrices, Quart. Journ. Math., Oxford (2), 7(1956), 138-143.

[3] W. V. PRKER, The characteristic roots of a imatrix, Duke Math. Journ. 3(1937), 484 A-7. 